\title{
Correlation between epidermal growth factor receptor tyrosine kinase inhibitor efficacy and circulating tumor cell levels in patients with advanced non-small cell lung cancer
}

\author{
This article was published in the following Dove Press journal: \\ OncoTargets and Therapy \\ 12 December 2016 \\ Number of times this article has been viewed
}

\author{
Wenjie $\mathrm{He}^{\prime}$ \\ Wenhui $\mathrm{Li}^{\prime}$ \\ Bo Jiang' \\ Li Chang' \\ Congguo Jin ${ }^{2}$ \\ Changlin Tu' \\ Yunfen $\mathrm{Li}^{\prime}$ \\ 'Department of Cadre's Medical \\ Oncology, Yunnan Tumor Hospital, \\ The Third Affiliated Hospital \\ of Kunming Medical University, \\ ${ }^{2}$ Department of Oncology Research \\ Institution, Kunming, People's \\ Republic of China
}

\begin{abstract}
Objective: The aim of this study was to investigate the correlation between the efficacy of epidermal growth factor receptor tyrosine kinase inhibitor (EGFR-TKI) and circulating tumor cell (CTC) levels in patients with advanced non-small cell lung cancer (NSCLC). The efficacy of EGFR-TKIs in reducing CTC counts in patients with advanced NSCLC was studied.

Patients and methods: A total of 66 patients with advanced NSCLC were enrolled and divided into two groups (those with high CTC counts and those with low CTC counts) based on the patients' median CTC counts. All the patients were treated with an EGFR-TKI, and the treatment efficacy and prognoses were compared.

Results: The treatment efficacies were 53.3\% (16/30) and 27.8\% (10/36) for the low CTC group and high CTC group, respectively, and this difference was statistically significant $(P<0.05)$. The median overall survival was 22.8 months (95\% confidence interval [CI]: 18.9-26.8 months) for the low CTC group and 18.3 months (95\% CI: 2.9-8.2 months) for the high CTC group. The median progression-free survival was 11.5 months (95\% CI: $8.1-15$ months) and 5.6 months (95\% CI: 2.9-8.2 months) for the low and high CTC groups, respectively, and the difference was statistically significant $(P<0.05)$.

Conclusion: The CTC count can be used as an index for predicting the EGFR-TKI effect on patients with advanced NSCLC. Efficacy and prognosis of EGFR-TKI treatment and CTC count were considered important, and the CTC count could be used to predict the efficacy of EGFRTKI treatment and prognosis of advanced NSCLC. The change in CTC expression levels can be used as an index for evaluating the prognosis of patients with advanced NSCLC.
\end{abstract}

Keywords: non-small cell lung cancer, circulating tumor cells, EGFR-TKI

\section{Introduction}

Lung cancer is the leading cause of cancer deaths worldwide, and non-small cell lung cancer (NSCLC) accounts for $85 \%$ of all lung cancer cases. ${ }^{1}$ Surgical treatment can obviously extend the survival time of patients with early-stage lung cancer. However, the early symptoms of lung cancer can hardly be noticed, without distinctive manifestation. Most patients are in locally advanced stage or have distant metastasis to the brain, bone, adrenal gland, liver, etc., at the time of initial diagnosis. If surgery cannot be conducted, the prognosis will be poor. The 5 -year survival rate of patients with stages I-IIIA lung cancer after excision is only $30 \%-40 \%$. $^{2}$ The overall 5 -year survival rate is $<15 \%$. Most patients die of recurrence of lung cancer and distant metastasis. ${ }^{3}$
Correspondence: Wenhui Li

Department of Cadre's Medical

Oncology, Yunnan Tumor Hospital,

The Third Affiliated Hospital of

Kunming Medical University, Kun

Zhou Road No 687, Kunming 650II8,

People's Republic of China

Tel/fax +8687I 81856562592

Email wenhuilidc@163.com 
As molecular targeted cancer therapy has improved, treatment for advanced NSCLC may involve new approaches. Epidermal growth factor receptor (EGFR) is the most studied cancer therapeutic target, and EGFR tyrosine kinase inhibitors (EGFR-TKIs) are an important option for targeting EGFR in treating cancer. Currently, EGFR-TKI is a first-line treatment option for patients with advanced NSCLC with EGFR mutations, but drug resistance limits the use of these compounds. Meanwhile, the detection of tumor and the monitoring of therapeutic process depend on imageological examination and serum tumor marker examination. However, the tumor size indicated by imaging is not in conformity with the grade malignancy or invasion and metastasis ability. ${ }^{3}$ Currently, a specific tumor marker with high sensitivity, which can dynamically evaluate the EGFR-TKI effect and timely find the recurrence and metastasis of tumor, is lacking. Therefore, a method that can dynamically evaluate the EGFRTKI effect, extend the survival time, and avoid excessive or delayed treatment is needed.

Cancer prognosis is related to tumor metastases that are believed to arise from circulating tumor cells (CTCs) that move into the peripheral blood. ${ }^{4,5}$ In addition, CTC counts are reported to be closely linked to lung cancer outcomes. ${ }^{6,7}$ In recent years, a minimally invasive approach was used to assess the efficacy of chemotherapy before and during cancer treatment, and CTC counts were found to be correlated with the efficiency of chemotherapy treatment in advanced NSCLC. ${ }^{8}$ However, in the field of EGFR-TKI therapy, no final conclusion has yet been reached on whether CTC count is effective in the evaluation and prognosis of EGFR-TKI effect. Therefore, we used CTCs as the research object, which were derived from the peripheral blood of patients with advanced NSCLC receiving EGFR-TKI treatment, and explored CTC count for evaluating the EGFR-TKI effect and prognosis in advanced NSCLC.

\section{Patients and methods Patient population}

This was a prospective phase II study, and 66 patients diagnosed with advanced NSCLC from October 2012 to May 2014 at the Yunnan Cancer Hospital were enrolled. Patients were required to be at least 18 years old and have an Eastern Cooperative Oncology Group Performance Status (PS) score of $\leq 3$, histologically or cytologically confirmed non-squamous stage IIIB NSCLC with pleural effusion or stage IV NSCLC, adequate organ function, and no history of prior systemic treatment of lung cancer. In this study, all of the 66 patients were
EGFR 19 exons or L858R exons mutation positive through EGFR gene mutation detected. But the anaplastic lymphoma kinase and KRAS mutations were not detected in the study. Patients with significant vascular disease or any serious cardiac condition were excluded. The study was approved by the Ethics Committee of Yunnan Cancer Hospital, and written informed consent was obtained from all the enrolled patients prior to any interventions. The CTC count was detected, and all the procedures performed in studies involving human participants were in accordance with the ethical standards of the institutional and/or national research committee and with the 1964 Declaration of Helsinki and its later amendments or comparable ethical standards.

\section{Patient treatments}

Patients were treated with one of the three EGFR-TKIs: gefitinib (250 mg/day, orally), erlotinib (150 mg/day, orally), or icotinib hydrochloride (125 mg thrice daily, orally). Therapy was continued for at least 1 month. The longest treatment course was 28 months, and the average course was 8 months.

\section{Sample collection and CTC measurements}

Blood was collected from patients prior to treatment and testing within $2 \mathrm{~h}$ after collection. Then, CTC count was measured according to the protocol. Briefly, mouse antihuman antibodies CD45-PC5, CK18-fluorescein isothiocyanate, and EGFR-phycoerythrin in addition to $\mathrm{NH} 4 \mathrm{Cl}$, hemolysin, Percoll, and FACS ${ }^{\text {TM }}$ Permeabilizing Solution 2 (Perm 2, 10×) reagents and a flow cytometry (EPICS XL) machine were purchased from Beckman Coulter. First, $20 \mu \mathrm{L}$ of CD45-PC5, $20 \mu \mathrm{L}$ of EGFR-phycoerythrin, and $20 \mu \mathrm{L}$ CK18-fluorescein isothiocyanate were added to $100 \mu \mathrm{L}$ of heparinized whole blood, and blood samples were kept at room temperature for $30 \mathrm{~min}$ in a dark room. Then, $\mathrm{NH}_{4} \mathrm{Cl}$ and hemolysin were added to lyse the cells. Cells in the samples were counted using a FACS machine after 15 min. CD45-CK18+ cells were considered CTCs. Counting was made per milliliter of blood. Cells were considered CTCs if they were negative for CD45 and presented with hyperchromatic nucleus, irregular shape, and high cytoplasm-nucleus ratio $(>0.5) .{ }^{9}$ Positive results for peripheral blood micrometastases were defined as CTCs $\geq 5 / 7.5 \mathrm{~mL}^{6}$

\section{Efficacy evaluation}

To evaluate the efficacy of clinical treatment, the World Health Organization's response assessment standards were used. ${ }^{10}$ 
Complete response (CR), partial response (PR), stable disease (SD), and disease progression (PD) were the categories of responses. CR and PR outcomes were considered results of effective treatment, and CR, PR, and SD outcomes were considered as having the disease under control. Progressionfree survival (PFS) was calculated from the first day of treatment until the date of PD, and overall survival (OS) was calculated from the first day of treatment until the date of death.

\section{Statistical analysis}

All analyses were conducted using SPSS software (Version 16.0; SPSS, Inc., Chicago, IL, USA). Individual variables were assessed using a paired rank sum test. Kaplan-Meier, $\log$ rank, and Cox multivariate regression analyses were used for survival analysis. $P<0.05$ was considered statistically significant.

\section{Results \\ Correlation between CTC count and clinical factors}

Patient characteristics are reported in Table 1. There were 40 males and 26 females, and the median age was 61 years (range, 41-78 years). Histologically, there were 58 patients with adenocarcinoma and 8 patients with squamous cell carcinoma. As per TNM staging, there were 4 cases of stage IIIB disease and 62 cases of stage IV disease. There were 44 cases with PS scores $0-1$ and 22 cases with PS scores $\geq 2$. We observed no significant association between CTC count

Table I Correlation between CTC count and clinical factors in patients with advanced NSCLC

\begin{tabular}{|c|c|c|c|c|}
\hline Clinical factors & $\mathbf{n}$ & CTC $(\bar{x} \pm s)$ & $t$ & $P$-value \\
\hline Gender & & & 2.573 & 0.11 \\
\hline Male & 40 & $133.9 \pm 249.6$ & & \\
\hline Female & 26 & $19.3 \pm 21.5$ & & \\
\hline Age (years) & & & 1.523 & 0.13 \\
\hline$\geq 60$ & 26 & $101.6 \pm 253.3$ & & \\
\hline$<60$ & 40 & $80.4 \pm 163.1$ & & \\
\hline Histologic type & & & 0.838 & 0.39 \\
\hline Squamous cell carcinoma & 8 & $92.8 \pm 168.8$ & & \\
\hline Adenocarcinoma & 58 & $116.6 \pm 208.6$ & & \\
\hline Clinical stage & & & 0.795 & 0.43 \\
\hline IIIB & 4 & $21.5 \pm 5.5$ & & \\
\hline IV & 62 & $93.1 \pm 207.6$ & & \\
\hline PS score & & & $1.35 \mathrm{I}$ & 0.18 \\
\hline $0-1$ & 44 & $93.5 \pm 217.3$ & & \\
\hline$\geq 2$ & 22 & $79.2 \pm|7|$ & & \\
\hline
\end{tabular}

Abbreviations: CTC, circulating tumor cell; NSCLC, non-small cell lung cancer; PS, performance status; $s$, standard deviation. and sex, age, histologic type, clinical stage, or PS score in any enrolled subject $(P>0.05$; Table 1$)$.

\section{CTC count and EGFR-TKI treatment efficacy}

CTC counts are reported in Table 2. In this study, the CTC expression value of the patients was 21.5-168. Based on our experimental data, according to the statistical method, 68.5, the median of CTC expression values of all patients, was used as the demarcation point. Patients with CTC expression values higher than the median were classified into high CTC expression group, and those with CTC expression values lower than the median were classified into low CTC expression group. The low CTC expression group had a response rate (RR) of 53.3\% (16/30) and a disease control rate (DCR) of $80 \%(24 / 30)$. The high CTC expression group had an RR of $27.8 \%(10 / 36)$ and a DCR of $44.4 \%$ (16/36). The difference between the two groups was statistically significant $(P<0.05)$. Treatment efficacy for each group is listed in Table 2. Efficacy was significantly higher in the low CTC group than in the high CTC group (Table 2).

\section{CTC counts before and after EGFR-TKI treatment}

Of the 66 patients, 40 patients were effectively treated $(\mathrm{CR}+\mathrm{PR}+\mathrm{SD}$ patients $)$, and $\mathrm{CTC}$ count in these patients was significantly different before and after treatment. The difference was statistically significant $(P=0.009)$. However, treatment was not effective in the other 26 patients (patients with PD), and CTC count in these patients was not significantly different between before and after treatment (Table 3). The difference was not statistically significant $(P=0.078$; Table 3$)$.

\section{Association between CTC count and survival}

The median OS was 20.6 months (95\% confidence interval [CI]: 17.9-23.4 months), and 1- and 3-year survival rates were $61.8 \%$ and $13.5 \%$, respectively. The median OS was 22.8 months (95\% CI: 18.9-26.8 months) in the low CTC group and 18.3 months (95\% CI: 14.8-21.9 months) in the high CTC group. In the low CTC group, the 1-year survival rate was $64.7 \%$, and that in the high CTC group was $56.2 \%$. The 3 -year survival rates were $20.6 \%$ in the low CTC group and $6.2 \%$ in the high CTC group. These survival differences were statistically significant $(P=0.01$; Figure 1). The total median PFS was 8.6 months $(95 \% \mathrm{CI}$ : 6.3-10.9 months), and the median PFS times were 11.5 months 
Table 2 Correlation between CTC count and efficacy of EGFR-TKI

\begin{tabular}{|c|c|c|c|c|c|c|c|c|c|c|c|c|}
\hline Group & $\mathbf{n}$ & CTC count $(\bar{x} \pm s)$ & CR & PR & SD & PD & RR (\%) & $X^{2}$ & $P$-value & DCR & $x^{2}$ & $P$-value \\
\hline Low count group & 30 & $21.5 \pm 8.3$ & 2 & 14 & 8 & 6 & 53.3 & 11.47 & 0.007 & 80 & 8.665 & 0.003 \\
\hline High count group & 36 & $118.3 \pm 168$ & 0 & 10 & 6 & 20 & 27.8 & & & 44.4 & & \\
\hline
\end{tabular}

Abbreviations: CR, complete response; CTC, circulating tumor cell; DCR, disease control rate; EGFR, epidermal growth factor receptor; PD, progressive disease; PR, partial response; RR, response rate; SD, stable disease; TKI, tyrosine kinase inhibitor; s, standard deviation.

(95\% CI: 8.1-15 months) and 5.6 months in the low CTC group and high CTC group (95\% CI: 2.9-8.2 months), respectively. These differences were statistically significant ( $P=0.005$; Figure 2). After treatment with EGFR-TKI, the RRs were $42.9 \%$ and $35.5 \%$ in the EGFR 19 exon mutation patients and EGFR L858R mutation patients, respectively, and there was no statistical difference $(P=0.21)$ between the two groups. Furthermore, the DCR rates were $60 \%$ in both the groups, with no statistical difference between the two groups $(P=0.54$; Table 4$)$.

\section{Discussion}

CTCs were first described based on their similarity to cancer cells. ${ }^{7}$ During malignant proliferation and development, tumor cells may be modified at the DNA or protein level to alter cellular phenotypes via epithelial-mesenchymal transition and invade the peripheral circulation to form metastatic CTCs. ${ }^{11}$

Chemotherapy is the mainstay treatment for advanced NSCLC, but RRs are poor. Research indicates that CTC counts negatively correlate with treatment efficacy. ${ }^{8}$ Oral EGFR-TKI treatment is an alternative option for patients with advanced NSCLC with unsuccessful primary chemotherapy. In tumor cells, EGFR is often mutated or overexpressed, resulting in tumor cell apoptosis inhibition and tumor growth promotion. ${ }^{12}$ Thus, EGFR-TKIs counteract this by blocking EGFR expression and promoting apoptosis. ${ }^{13}$ Because EGFR-TKI treatment increases PFS and OS times in patients with EGFR mutations, EGFR-TKI is a first-line treatment for patients with advanced NSCLC with EGFR mutations. However, drug resistance limits the use of EGFR-TKIs,

Table 3 Changes in CTC count before and after EGFR-TKI treatment

\begin{tabular}{llllll}
\hline Group & $\mathbf{n}$ & $\begin{array}{l}\text { Before } \\
\text { treatment }\end{array}$ & $\begin{array}{l}\text { After } \\
\text { treatment }\end{array}$ & $\boldsymbol{t}$ & P-value \\
\hline Effective & 40 & $19.4 \pm 20.1$ & $14.8 \pm 12.3$ & 2.46 & 0.009 \\
Noneffective & 26 & $195.4 \pm 292.9$ & $107.6 \pm 227$ & 1.55 & 0.078 \\
\hline
\end{tabular}

Abbreviations: CTC, circulating tumor cell; EGFR, epidermal growth factor receptor; TKI, tyrosine kinase inhibitor.

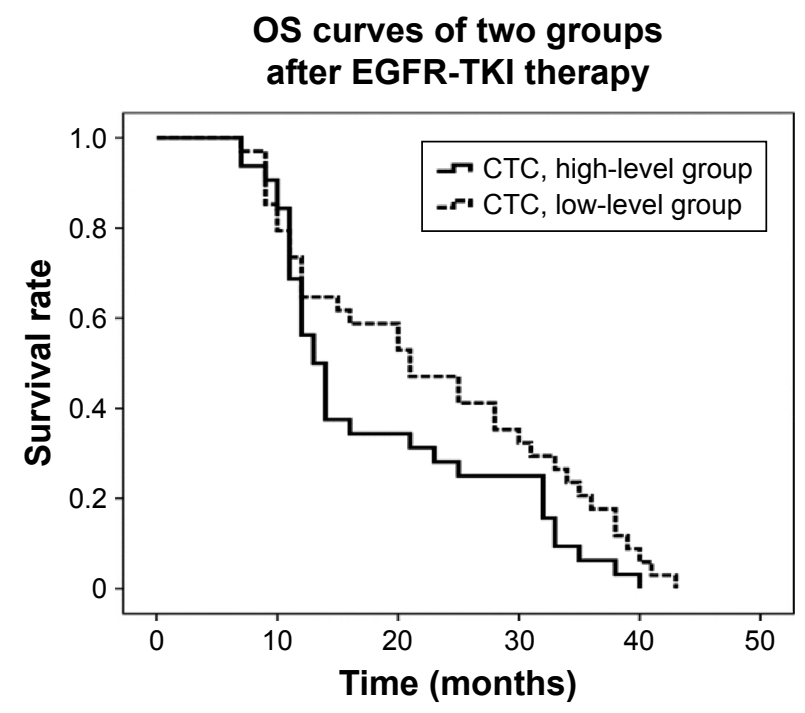

Figure I Correlation between CTC count and survival in patients with advanced NSCLC.

Abbreviations: CTC, circulating tumor cell; EGFR, epidermal growth factor receptor; NSCLC, non-small cell lung cancer; OS, overall survival; TKI, tyrosine kinase inhibitor.

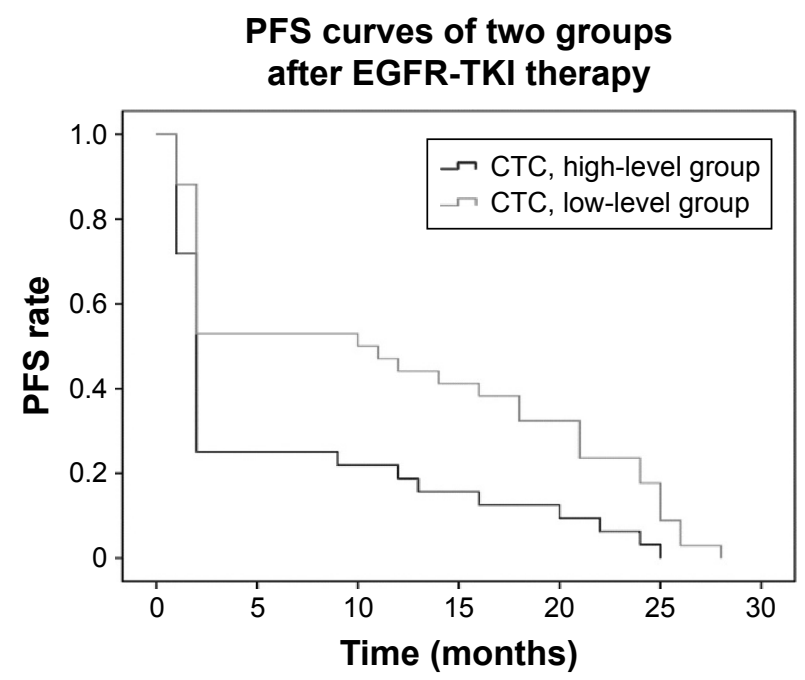

Figure 2 Correlation between CTC count and PFS in patients with advanced NSCLC.

Abbreviations: CTC, circulating tumor cell; EGFR, epidermal growth factor receptor; NSCLC, non-small cell lung cancer; PFS, progression-free survival; $\mathrm{TKI}$, tyrosine kinase inhibitor. 
Table 4 Different mutation state of EGFR showing the different curative effect of EGFR-TKI

\begin{tabular}{|c|c|c|c|c|c|c|c|c|c|c|c|c|}
\hline Group & $\mathbf{n}$ & CTC count $(\bar{x} \pm s)$ & CR & PR & SD & PD & $\mathbf{R R}(\%)$ & $x^{2}$ & $P$-value & DCR & $x^{2}$ & $P$-value \\
\hline EGFR 19 exons & 35 & $98.5 \pm 121.1$ & 2 & 13 & 6 & 14 & 42.9 & 4.38 & 0.21 & 60 & 2.17 & 0.54 \\
\hline EGFR L858R exons & 31 & $108.2 \pm|4| .3$ & 0 & 11 & 8 & 12 & 35.5 & & & 61.3 & & \\
\hline
\end{tabular}

Abbreviations: CR, complete response; CTC, circulating tumor cell; DCR, disease control rate; EGFR, epidermal growth factor receptor; PD, progressive disease; PR, partial response; RR, response rate; SD, stable disease; TKI, tyrosine kinase inhibitor; s, standard deviation.

and the amplification of T790M, a point mutation in EGFR exon 20 , is believed to mediate this resistance. This mutation can increase the affinity between EGFR and ATP. Because of this, reversible TKIs cannot easily inhibit the phosphorylation of the EGFR and mesenchymal-epithelial transition (MET) tyrosine kinase growth factor receptor families. Hepatocyte growth factor receptor is an MET family receptor tyrosine kinase, and it is relevant to cellular proliferation. MET gene amplification can activate the ErbB3/PI3K/AKT signaling pathway and elicit resistance to EGFR kinase inhibitors. ${ }^{14}$ Thus, knowing whether EGFR-TKIs will be efficacious is important for treatment and disease prognosis.

Recently, CTC measurements and peripheral blood gene expression data have been used to guide chemotherapy. Tumor cells can be found in peripheral blood, body fluids, and pleural effusions, and as such, CTCs can serve as proxies for cancerous tissues to test tumor-associated gene or protein expression. However, CTC genomic testing as a replacement for solid tumor genomic detection is unclear. Punnoose et $\mathrm{al}^{15}$ reported that genomic expression in CTCs in EGFR-TKItreated patients with NSCLC was highly consistent with genomic expression in primary tumor tissue. Maheswaran et $\mathrm{al}^{16}$ reported that EGFR, EGFR T790M, MET, and other gene mutations in CTCs can be used to evaluate treatment efficacy and prognosis in patients with NSCLC.

There are very few CTCs in the blood of patients with advanced NSCLC, especially during early stages. For this reason, it is very important to establish an accurate, reliable, and reproducible technical method for detecting CTCs from peripheral blood. Common CTC detection methods include Cell Search ${ }^{\mathrm{TM}}$ systems, reverse transcription polymerase chain reaction, CTC chip, and flow cytometry. These technologies can significantly improve detection sensitivity and specificity of CTC testing. Flow cytometry is a high-speed cell analysis and sorting technology, which uses fluorescent antibodies to stain and analyze tumor cells. Flow cytometry can perform multiparameter analysis of the same cell, which may reveal physical and chemical characteristics of the cells. Because it is simple, reliable, and fast, flow cytometry was selected as the main method for the detection of CTCs.
In this study, CTCs from the peripheral blood of patients with advanced NSCLC were counted using flow cytometry. Data indicate that EGFR-TKIs were more efficacious for patients with low CTC counts, and better DCRs were achieved in these patients. CTC counts obtained before and after treatment did not differ significantly. These data agree with the published reports that support the use of CTC counts for predicting treatment outcomes. ${ }^{16,17}$

Significantly longer median overall, 1-year, and 3-year survival rates are reported in this study for the low CTC group than for the high CTC group ( $P=0.01)$. The median PFS was also significantly higher in the low CTC group $(P=0.005)$. These data were explained by a greater therapeutic efficacy using EGFR-TKIs and better disease control. This is why CTC counts are closely related to the prognosis of patients with advanced NSCLC treated with EGFR-TKI therapy. As such, CTC counts may be used for making treatment decisions. Our study sample size was small, and therefore further studies with a large sample size are needed to confirm these results. CTC can also be used as a carrier line for genomic testing to confirm that CTCs can serve as predictors for the efficacy and prognosis of EGFR-TKI therapy in patients with advanced NSCLC.

\section{Conclusion}

The CTC level can be used as an index for predicting the EGFR-TKI effect in patients with advanced NSCLC. Efficacy and prognosis of EGFR-TKI treatment and CTC count were considered important, and the CTC count could be used to predict the efficacy of EGFR-TKI treatment and prognosis of advanced NSCLC. The change in CTC expression levels can be used as an index for evaluating the prognosis of patients with advanced NSCLC.

\section{Acknowledgment}

This work was supported by the Department of Education Yunnan province (2016ZDX059).

\section{Author contributions}

Performed the molecular genetic studies, participated in sequence alignment, and drafted the manuscript: WH. 
Performed the immunoassays: BJ and LC. Participated in sequence alignment: CT and YL. Participated in study design and performed the statistical analysis: CJ. Conceived the study, participated in design and coordination, and helped draft the manuscript: WL. All authors contributed toward data analysis, drafting and critically revising the paper and agree to be accountable for all aspects of the work.

\section{Disclosure}

The authors report no conflicts of interest in this work.

\section{References}

1. Torre LA, Siegel RL, Jemal A. Lung cancer statistics. Adv Exp Med Biol. 2016;893:1-19.

2. Siegel R, Naishadham D, Jemal A, Perelson AS, Lenardo MJ, Speirs C. Cancer statistics, 2013. CA Cancer J Clin. 2013;63(1):11-30.

3. Stojiljkovic D, Santrac N, Goran M, et al. Factors related to local recurrence of non small cell lung cancer and its operability. J BUON. 2016; 21(1):221-226.

4. Yamamoto O, Takahashi H, Hirasawa M, et al. Surfactant protein gene expressions for detection of lung carcinoma cells in peripheral blood. Respir Med. 2013;99(9):1164-1174.

5. Krebs MG, Sloane R, Priest L, et al. Evaluation and prognostic significance of circulating tumor cells in patients with non-small cell lung cancer. J Clin Oncol. 2013;29(12):1556-1563.

6. Hou JM, Greystoke A, Lancashire L, et al. Evaluation of circulating tumor cells and serological cell death biomarkers in small cell lung cancer patients undergoing chemotherapy. Am J Pathol. 2012;175(2): 808-816.

7. Hofman V, Ilie MI, Long E, et al. Detection of circulating tumor cells as a prognostic factor in patients undergoing radical surgery for non-small-cell lung carcinoma: comparison of the efficacy of the CellSearch Assay ${ }^{\mathrm{TM}}$ and the isolation by size of epithelial tumor cell method. Int $J$ Cancer. 2013;129(7):1651-1660.
8. Krebs MG, Sloane R, Priest L, et al. Evaluation and prognostic significance of circulating tumor cells in patients with non-small cell lung cancer. J Clin Oncol. 2004;29(12):1556-1563.

9. Buim ME, Fanelli MF, Souza VS, et al. Detection of KRAS mutations in circulating tumor cells from patients with metastatic colorectal cancer. Cancer Bio Ther. 2015;16(9):1289-1295.

10. Kuiper JL, Heideman DA, Würdinger T, Grünberg K, Groen HJ, Smit EF. Rationale and study design of the IRENE-trial (NVALT-16): a phase II trial to evaluate iressa rechallenge in advanced NSCLC patients with an activating EGFR mutation who responded to an EGFRTKI used as first-line or previous treatment. Clin Lung Cancer. 2015; 16(1):60-66.

11. Langley RR, Fidler IJ. The seed and soil hypothesis revisited - the role of tumor-stroma interactions in metastasis to different organs. Int J Cancer. 2013;128(11):2527-2535.

12. Ragusa M, Vannucci J, Ludovini V, et al. Impact of epidermal growth factor receptor and KRAS mutations on clinical outcome in resected non-small cell lung cancer patients. Am J Clin Oncol. 2014;37(4): 343-349.

13. Tamura K, Okamoto I, Kashii T, et al. Multicentre prospective phase II trial of gefitinib for advanced non-small cell lung cancer with epidermal growth factor receptor mutations: results of the West Japan Thoracic Oncology Group trial (WJTOG0403). Br J Cancer. 2008;98(5): 907-914.

14. Nguyen KS, Kobayashi S, Costa DB. Acquired resistance to epidermal growth factor receptor tyrosine kinase inhibitors in non-small-cell lung cancers dependent on the epidermal growth factor receptor pathway. Clin Lung Cancer. 2009;10(4):281-289.

15. Punnoose EA, Atwal S, Liu W, et al. Evaluation of circulating tumor cells and circulating tumor DNA in non-small cell lung cancer: association with clinical endpoints in a phase II clinical trial of pertuzumab and erlotinib. Clin Cancer Res. 2012;18(8):2391-2401.

16. Maheswaran S, Sequist LV, Nagrath S, et al. Detection of mutations in EGFR in circulating lung-cancer cells. $N$ Engl J Med. 2008;359(4): 366-377.

17. Nagrath S, Sequist LV, Maheswaran S, et al. Isolation of rare circulating tumour cells in cancer patients by microchip technology. Nature. 2007;450(7173):1235-1239.
OncoTargets and Therapy

\section{Publish your work in this journal}

OncoTargets and Therapy is an international, peer-reviewed, open access journal focusing on the pathological basis of all cancers, potential targets for therapy and treatment protocols employed to improve the management of cancer patients. The journal also focuses on the impact of management programs and new therapeutic agents and protocols on

\section{Dovepress}

patient perspectives such as quality of life, adherence and satisfaction. The manuscript management system is completely online and includes a very quick and fair peer-review system, which is all easy to use. Visit http://www.dovepress.com/testimonials.php to read real quotes from published authors. 